\title{
Cardiac Manifestations in Systemic Lupus Erythematosus: A Case Report and Review of the Literature
}

\author{
Alexandra Kreps M.D., Karen Paltoo B.A., Isabel McFarlane M.D* \\ Department of Medicine, Division of Rheumatology, State University of New York, \\ Downstate Medical Center/Health + Hospitals Kings County Brooklyn, NY 11203 USA \\ *Corresponding author: Isabel.McFarlane@downstate.edu
}

Received August 10, 2018; Revised September 15, 2018; Accepted October 10, 2018

\begin{abstract}
Background: Systemic Lupus Erythematosus (SLE) is a chronic autoimmune disease with a wide range of clinical features and variable clinical course. SLE tends to affect women during childbearing years and is characterized by multi-organ involvement. Cardiac complications in SLE, which have been described to occur in about $50 \%$ of the cases, contributes to significant morbidity and mortality in this population. We describe a patient with SLE and established lupus nephritis who subsequently developed cardiac manifestations including valvular abnormalities, arrythmia and end stage heart failure. The clinical features, work up and management will be discussed. Case presentation: A 35 year-old African American woman diagnosed with SLE in her twenties presented to our hospital for evaluation of shortness of breath. After SLE diagnosis, the patient had been prescribed hydrochloroquine and low dose steroids for joint and skin manifestations. Four years after initial presentation, she developed biopsy proven lupus nephritis for which standard induction therapy was administered. She was placed on maintenance immunosuppression with stable renal function. On admission, the patient's symptoms included dyspnea on exertion, chest pain, palpitations, and a non-productive cough. Initial evaluation identified atrial fibrillation and new onset of heart failure given elevated brain natriuretic peptide (BNP) levels and left ventricular ejection fraction (EF) of $15 \%$ by echocardiogram. Cardiac catheterization revealed global hypokinesis and non-obstructive coronary artery disease (CAD). The patient was deemed not a suitable candidate for cardiac transplant and was offered a life vest as bridging to an implantable cardioverter (ICD). Twenty-four months after discharge, the patient continued to be managed medically and has not had any subsequent hospitalizations. Conclusion: Cardiac complications, reported in about $50 \%$ of SLE patients, are associated with high morbidity and mortality. Pericarditis is the most common, however conduction defects, valvular damage and heart failure are also observed among SLE patients. The pathogenesis of cardiac involvement seems to be multifactorial. The management of heart failure in SLE entails medical therapy and implantable device use. Further research is needed to explore new options to arrest the development and progression of cardiac disease among lupus patients.
\end{abstract}

Keywords: systemic lupus erythematosus, coronary heart disease, coronary artery disease, congestive heart failure, end-stage heart failure, cardioverter placement, cardiac resynchronization therapy

Cite This Article: Alexandra Kreps M.D., Karen Paltoo B.A., and Isabel McFarlane M.D., "Cardiac Manifestations in Systemic Lupus Erythematosus: A Case Report and Review of the Literature." American Journal of Medical Case Reports, vol. 6, no. 9 (2018): 180-183. doi: 10.12691/ajmcr-6-9-3.

\section{Introduction}

Systemic Lupus Erythematosus (SLE) is a chronic autoimmune disease that is characterized by inflammation and damage to multiple organ systems [1]. Cardiac involvement can be seen in over $50 \%$ of lupus patients, affecting all structural components of the heart. The postulated mechanisms underlying the pathogenesis of cardiac involvement including risk factors and genetic predisposition will be discussed.

\section{Case Presentation}

A 35 year-old African American woman was diagnosed with SLE at the age of 22 when she presented with arthralgias, subacute cutaneous lupus, and leukopenia. Her disease was controlled with hydrochloroquine and low dose steroids. Four years after diagnosis, the patient developed proteinuria and underwent renal biopsy which demonstrated lupus nephritis class IV and V. She received pulse steroids followed by low dose cyclophosphamide. 
Rituximab was also administered but was stopped due to pregnancy. After delivery, the patient was placed on mycophenolate mofetil (MMF). However, MMF was discontinued in view of a second pregnancy. The patient was able to carry two full term pregnancies while on hydrochloroquine (HCQ), low dose prednisone, and azathioprine (AZA) which were maintained on a chronic basis. Her renal function stabilized with a creatinine of 1.9 and proteinuria of 800 grams.

One year prior, the patient had developed shortness of breath and a new heart murmur was found. An echocardiogram revealed a free-floating mass in the left atrium with moderate mitral valve regurgitation. A trial of warfarin was given and after three months since the size of the mass remained unchanged, atrial myxoma rather than atrial thrombus was suspected. Anti-phospholipid antibodies were negative. Patient underwent atrial myxoma removal and mitral valve annuloplasty. The post-operative course was complicated by new onset atrial fibrillation and warfarin was initiated. The patient stabilized and continued her regimen of low dose steroids, HCQ and AZA.

The patient presented to our institution for complaints of dyspnea, chest pain, palpitations, non-productive cough, fevers and chills for several months, that had worsened over the last few days. On physical exam, she had rales and peripheral edema. Significant labs revealed anemia, elevated creatinine, proteinuria, brain natriuretic peptide $>20,000 \mathrm{pg} / \mathrm{mL}$ and low complement levels (see Table 1). Echocardiogram and left heart catheterization demonstrated global hypokinesis with an EF of 15\%, and non-obstructive coronary artery disease. As her renal function declined, patient was initiated on hemodialysis.

Table 1. Laboratory Data

\begin{tabular}{|l|c|c|}
\hline Serum & Patient & Reference Range \\
\hline WBC (K/uL) & 6.26 & $4.5-10.9$ \\
\hline RBC (M/uL) & 3.48 & $4.2-5.4$ \\
\hline Hemoglobin (g/dL) & 9.7 & $12.0-16.0$ \\
\hline Hematocrit (\%) & 30.8 & $37.0-47.0$ \\
\hline Platelets (K/uL) & 267 & $130-400$ \\
\hline & & \\
\hline Sodium (mmol/L) & 136 & $136-146$ \\
\hline Potassium (mmol/L) & 4.1 & $3.5-5.0$ \\
\hline Chloride (mmol/L) & 99 & $98-106$ \\
\hline Blood Urea Nitrogen (mg/dL) & 40 & $6-20$ \\
\hline Creatinine (mg/dL) & 6.45 & $0.4-1.2$ \\
\hline Calcium (mg/dL) & 8.7 & $8.4-10.3$ \\
\hline Total Protein (g/dL) & 5.2 & $6.0-8.5$ \\
\hline Albumin (g/dL) & 2.82 & $2.8-5.7$ \\
\hline Aspartate Aminotransferase (U/L) & 20 & $10-35$ \\
\hline Alanine Aminotransferase (U/L) & 8 & $0-31$ \\
\hline Alkaline Phosphatase (U/L) & 80 & $25-125$ \\
\hline Total Bilirubin & 0.60 & $0.0-1.2$ \\
\hline Glucose (mg/dL) & 101 & $70-99$ \\
\hline & & \\
\hline ESR(mm/h) & 31 & $0-20$ \\
\hline CRP(mg/dL) & 11.4 & $1.0-4.0$ \\
\hline Complement C3 (mg/dL) & 44.8 & $86-184$ \\
\hline Complement C4 (mg/dL) & $<8.0$ & $20-58$ \\
\hline Brain natriuretic peptide (pg/mL) & $>20,000$ & $<100$ \\
\hline Antinuclear Antibiodies & Positive & $.00-.70$ \\
\hline Anti-ds DNA & Positive & $.00-10.00$ \\
\hline & & \\
\hline
\end{tabular}

After several days of medical optimization, a life vest was placed as bridging to implantable cardioverter placement (ICD). Cardiothoracic surgery evaluation deemed the patient not a suitable candidate for heart transplant given end stage renal disease requiring hemodialysis.

Upon discharge, she continues on medical management as an outpatient, with dialysis three times a week. She has had no subsequent hospitalizations for heart failure or lupus flairs within the past 24 months and continues to be followed in rheumatology, renal and cardiology clinics.

This patient's family history included two sisters with lupus and a third sister with mixed connective tissue disease and Raynaud's phenomenon. One of the sisters developed lupus nephritis and an atrial thrombus.

\section{Discussion}

SLE is an inflammatory autoimmune disorder that affects most commonly women in childbearing years, leading to involvement of almost any organ system [1]. Overall, SLE may affect $40-200$ per 100,000 persons, with predominance in the Black population. Throughout the course of the disease, cardiac manifestations can develop in more than $50 \%$ of patients of lupus patients, leading to increased mortality [1]. The risk of myocardial infarction is up to nine times greater among SLE patients compared to the general population and 50 times greater when compared to healthy women only $[3,4]$. However, the pathogenesis of cardiac involvement is largely unknown with multiple factors playing a role [2]. Our patient's heart failure presentation was likely the result of a combination of innate immunity disarray, genetics and environmental factors.

Similar to patients with diabetes mellitus, patients with SLE have features of accelerated atherosclerosis that may appear early in the course of the disease [5]. As the disease progresses, patients face increased risk of coronary heart disease (CHD) and stroke [5]. Abnormal lipid and lipoproteins levels commonly seen in SLE, may play a key role in accelerated atherosclerosis as patients with active SLE had higher levels of cholesterol and triglycerides, along with lower levels of high-density lipoproteins [6]. Increased endothelial dysfunction may also play a role in atherogenesis and is associated with carotid artery intima-media thickness, an early marker of atherosclerosis [7]. Autoantibodies also mediate cardiac damage, as deposition of immunoglobulins and C3 in the pericardium may lead to inflammatory changes in the heart [8]. In patients with antiphospholipid (APL) syndrome and SLE, APL antibodies may play a role in thrombotic events, as well as lesions affecting the heart valves, endocardium, and papillary muscles [8].

Traditional risk factors for CHD do not explain impaired endothelial function or the number of increased cardiovascular events seen in SLE [3,7]. Patients with SLE have increased risk for cardiovascular events beyond the traditional Framingham risk factors [3]. Thus, chronic inflammation has been postulated to play a role in accelerated atherosclerosis. Furthermore, chemotherapies like cyclophosphamide may contribute to the development of cardiac disease $[9,10]$. Additionally, research has shown that genetic markers may also contribute to specific SLE complications $[11,12]$. 
Cardiac involvement in SLE can affect the pericardium, myocardium, and endocardium. Pericarditis, the most studied, is estimated to affect $25 \%$ of SLE patients at disease onset or during relapses [8,13]. Asymptomatic pericardial effusion has been reported in up to $40 \%$ [8]. Myocarditis is rare, but can lead to arrhythmias, ventricular dysfunction, dilated cardiomyopathy, and heart failure. Libman-Sacks endocarditis has been described to affect 1 in 10 patients in some series and is recognized by verrucous growth occurring on the valve leaflets, papillary muscles and endocardium [8,14]. Valvular thickening, vegetations, and dysfunction have also been described in SLE with the tricuspid valve being commonly involved [15].

Similarly to renal transplant being an option for end-stage renal disease due to lupus nephritis, cardiac transplant is a viable option for SLE patients in heart failure [17]. However, cardiac transplantation is not feasible for all cases due to long waiting lists, difficulty finding matches, and exclusion criteria. Therefore, it is imperative that other options, such as cardiac resynchronization therapy (CRT) devices and ICDs, be offered to these patients. CRT may even lessen cardiac inflammation by reducing inflammatory cytokines [17]. To date, there is insufficient data on the utilization and long-term outcomes of CRT and ICD usage among SLE patients.

Future treatments that aim to improve the endothelial function in patients may also decrease the risk of CAD development, however, much is unknown about the mechanism behind this dysfunction [7]. For example, the drug canakinumab, which targets the proinflammatory cytokine interleukin-1ß (IL1ß), can possibly help prevent endothelial dysfunction by preventing monocyte and leukocyte adhesion to vascular endothelial cells [21]. Therefore, future studies may focus on the pathogenesis of endothelial dysfunction by exploring proinflammatory cytokines like IL1ß to develop other targeted treatments to prevent progression to CAD in the setting of SLE. Furthermore, this type of treatment was successful in primary systemic vasculitis, where suppression of inflammation restored vascular endothelial function [18].

Finally, this patient's family cluster also highlights the role that genetics plays in the pathogenesis of SLE and its cardiac manifestations. Many genetic studies have begun exploring various markers linked to specific disease manifestations in SLE. Interleukin 19 (IL-19) risk locus was found in a cluster of genes that code for cytokines IL19 and IL10 [19]. Patients with SLE and the IL19 risk allele had a higher incidence of MI, and even when adjusting for the known CVD risk factors, the IL-19 risk allele association was consistent [19]. Therefore, the IL-19 risk allele appears to be an independent risk factor for cardiovascular disease among patients with SLE [19]. Overall, more research into the various components of cardiac complications of SLE must be explored to further guide disease management.

\section{Conclusion}

About $50 \%$ of SLE patients can develop cardiac complications which carries a heightened morbidity and mortality. Although, all the three layers of the heart could be affected, the pericarditis is the most commonly observed among SLE patients. SLE disease activity, chronic inflammation, genetic predisposition and treatment adverse effects have been postulated as important players in the pathogenesis of cardiac involvement. The management of heart failure in SLE is based on the current knowledge available for the management of heart failure in the general population, including medical management and CRT and ICD utilization. Heart transplant option should be explored for all end stage heart disease SLE patients. Research is ongoing aiming to find pathways to control of inflammation in SLE which appears to be key in arresting the development and progression of cardiac disease.

\section{Acknowledgements}

This work is supported in part by Dr. Moro O. Salifu's efforts through NIH Grant \# S21MD012474.

\section{References}

[1] Jain, D., Halushka, M.K. Cardiac Pathology of Systemic Lupus Erythematosus. J Clin Pathol (2009). 62: 584-592.

[2] Kim C.H., Al-Kindi S.G., Jandali B., Askari A.D., Zacharias M., Oliveira G.H. Incidence and risk of heart failure in systemic lupus erythematosus. Heart. 2016; 103(3): 227-233.

[3] Jonsson, H., Nived O., Sturfelt G. Outcome in systemic lupus erythematosus: a prospective study of patients from a defined population. Medicine (Baltimore) 1989; 68: 141-50.

[4] Manzi S, Meilahn EN, Rairie JE, Conte CG, Medsger TA Jr, Jansen-McWilliams L, et al. Age-specific incidence rates of myocardial infarction and angina in women with systemic lupus erythematosus: comparison with the Framingham study. Am J Epidemiol 1997; 145: 408-15.

[5] Esdaile JM, Abrahamowicz M, Grodzicky T, Li Y, Panaritis C, du Berge $\mathrm{R}$, et al.Traditional Framingham risk factors fail to fully account for accelerated atherosclerosis in systemic lupus erythematosus. Arthritis Rheum 2001; 44: 2331-7.

[6] Posadas-Romero C, Torres-Tamayo M, Zamora-Gonzalez J, Aguilar-Herrera BE, Posadas-Sanchez R, Cardosa-Saldana G, Ladron de Guevara G, Solis-Vallejo E, El Hafidi M. High insulin levels and increased low-density lipoprotein oxidizability in pediatric patients with systemic lupus erythematosus. Arthritis Rheum. 2004; 50: 160-165.

[7] El - Magadmi M, Bodill H, Ahmad Y, Durrington PN, Mackness M, Walker M, et al.Systemic lupus erythematosus: an independent risk factor for endothelial dysfunction in women. Circulation 2004 110: 399-404.

[8] Tincani, A., Rebaioli, C.B., Taglietti,M., Shoenfeld, Y., Heart Involvement in systemic lupus erythematosus, anti-phospholipid syndrome and neonatal lupus, Rheumatology 2006, vol 45 (8-3).

[9] Atalay, F., Gulmez, O., and Ugurlu, A.O. Cardiotoxicity following cyclophosphamide therapy: a case report. Journal of Medical Case Reports 2014. 8: 252.

[10] Wu, H., Zeng, J., Yin, J., Peng, Q., Zhao, M., Lu, Q. Organspecific biomarkers in lupus. Autoimmunity reviews. 2017. Vol 16, Issue 4. 391-397.

[11] Leonard, D., Svenungusson, E., Sandling, J.K, et al. Coronary heart disease in systemic lupus erythematosus is associated with interferon regulatory factor- 8 gene variants. Circ Cardiovasc Genet. 2013 Jun; 6(3): 255-63.

[12] Ciccacci C, Perricone C, Ceccarelli F, et al. A Multilocus Genetic Study in a Cohort of Italian SLE Patients Confirms the Association with STAT4 Gene and Describes a New Association with HCP5 Gene. PLoS ONE. 2014; 9(11).

[13] Doria A, Iaccarino L, Sarzi-Puttini P, et al. Cardiovascular involvement in systemic lupus erythemathosus, Lupus, 2005, vol. 14 (pg. 683-6). 
[14] Moyssakis I., Tektonidou M.G., Vasilliou V.A. Libman-Sacks endocarditis in systemic lupus erythematosus: prevalence, associations, and evolution. Am J Med. 2007; 120: 636-642.

[15] Hojnik M, George J, Ziporen L, Shoenfeld Y. Heart valve involvement (Libman-Sacks endocarditis) in the antiphospholipid syndrome, Circulation, 1996, vol. 93 (pg. 1579-87).

[16] Cervera R, Khamashta MA, Font J, et al. Morbidity and mortality in systemic lupus erythematosus during a 5 year period: a multicenter prospective study of 1000 patients, Medicine, 1999, vol. 78 (pg. 167-75).

[17] Ashrafi, R., Garg, P., McKay, E., Gosney, J., Chuah, S., and Davis G. Aggressive cardiac involvement in systemic lupus erythematosus: a case report and a comprehensive literature review. Cardiol Res Pract. 2011; 2011: 578390.
[18] Raza K, Thambyrajah J, Townend JN, et al. Suppression of inflammation in primary systemic vasculitis restores vascular endothelial function: lessons for atherosclerotic disease? Circulation. 2000; 102: 1470-1472.

[19] Leonard D, Svenungsson E, Dahlqvist J, et al. Ann Rheum Dis 2018;77: 1063-1069.

[20] EJ, Dellsperger KC. Cardiorenal Syndrome: The Clinical Cardiologists' Perspective. Cardiorenal Medicine. 2011; 1(1): 13-22.

[21] Ridker, PM, Everett, BM, Thuren, T, MacFadyen, JG, Chang, WH, Ballantyne, C., Fonseca, F., Nicolau, J., Koenig, W, Anker, $\mathrm{SD}$ et al. Antiinflammatory therapy with Canakinumab for atherosclerotic disease. N Engl J Med, 377 (2017), pp. 1119-1131. 